EPJ Web of Conferences 110,01064 (2016)

DOI: 10.1051/epjconf/201611001064

(C) Owned by the authors, published by EDP Sciences, 2016

\title{
APPLICATION OF A CONVECTIVE-CONDUCTIVE HEAT TRANSFER MODEL IN THE HEAT LOSS ANALYSIS OF A HEAT PIPELINE UNDER FLOODING CONDITIONS
}

\author{
V.Yu. Polovnikov ${ }^{1, \text { a }}$, N.V. Razumov ${ }^{1}$ \\ ${ }^{1}$ National Research Tomsk Polytechnic University, 634050 Tomsk, Russia
}

\begin{abstract}
This paper describes the numerical modeling of a convective-conductive heat transfer the area placing of a heat pipeline under flooding conditions. We have established that the heat loss of a heat pipeline under flooding conditions increases in the range from 1.5 to $64.3 \%$, depending on the volume fraction of water in the insulation structure.
\end{abstract}

\section{Introduction}

There are many publications on research to develop and improve the efficiency of heating systems in the modern scientific literature [1-11]. Publication of a heat loss of heat pipeline [7-11] is very important.

One of the promising approaches to study of modes of heat pipeline in the real application conditions is a numerical simulation. This makes it possible to take account of different effects and processes which lead to an intensification of heat and mass transfer in under consideration systems.

The aim of the present paper is a mathematical modeling of thermal regimes and numerical analysis of a heat loss of a heat pipeline under flooding conditions with use a convective-conductive heat transfer model.

\section{Problem statement}

We consider a typical underground channel heat pipeline. Pipes insulated with a mineral wool and a protective layer [11].

An underground channel heat pipeline is operated under flooding conditions. Figure 1 shows a scheme of decision domain.

For the domain under consideration (Fig. 1) we solve a 2D linear and stationary problem of convective-conductive heat transfer.

Formulating the problem, we used the following assumptions:

1. The heat transfer processes in the internal and the external environment are disregarded.

2. Thermophysical characteristics of materials used in the analysis are constant and known values.

3. There is an ideal thermal contact conditions at the boundaries.

a Corresponding author: polov@tpu.ru

This is an Open Access article distributed under the terms of the Creative Commons Attribution License 4.0, which permits unrestricted use, distribution, and reproduction in any medium, provided the original work is properly cited. 
4. The heat in the solid decision domain (Fig. 1) is transferred only by conduction.

The listed assumptions, on the one hand, do not impose constrains of principle on the physical model of the system (Fig. 1), but, on the other hand, allow one to simplify in a certain manner the algorithm and method for solving the posed problem.

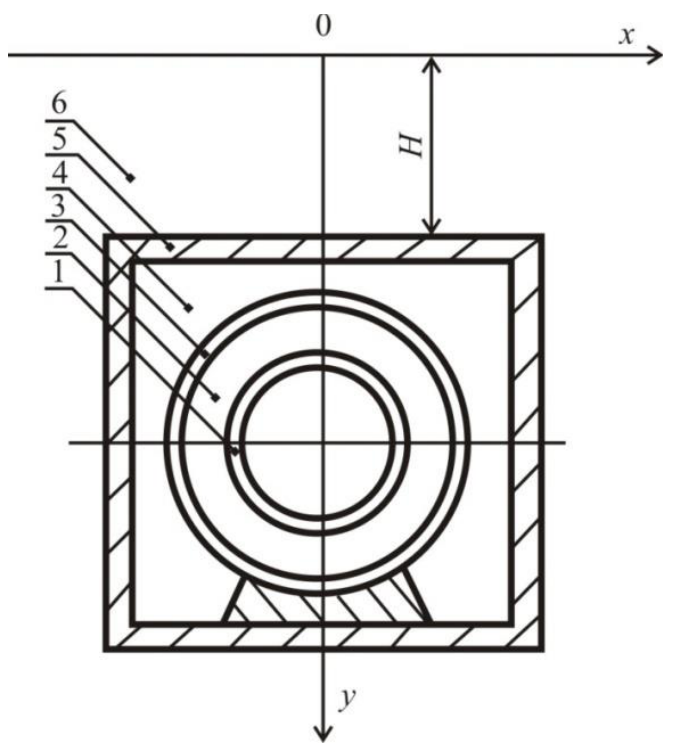

Figure 1. A scheme of decision domain: 1 - metal wall of the heat pipeline; 2 - insulating layer; 3 - protective layer; 4 -cavity of channel; 5 - channel; 6 -ground; $H$-distance from the ground surface to the upper part of channel.

\section{Mathematical model and method of solution}

The mathematical model is completely similar to that described in [10]. The system of equations [10] was solved by the finite element method [12], using the Galerkin approximation [13]. The investigations were carried out on a nonuniform finite-element mesh. The number of elements was chosen from conditions of convergence of solution, the mesh was made denser by the Delaunay method $[12,13]$.

In spite of the fact that in statement of the problem it was assumed to use an infinite-size domain, in the numerical analysis of heat loss we used a calculation domain of final size. The sizes of the calculation domain were chosen on the basis of a series of preliminary numerical experiments in such a manner that the relative change of the temperature gradients at the domain boundary does not exceed $0.5 \%$.

\section{Initial data}

The analysis was carried out for a pipeline with a diameter of nominal bore of $600 \mathrm{~mm}$; the pipeline was manufactured from steel 10 (thickness $6 \mathrm{~mm}$ ) with thermal insulation from a mineral wool $(70$ $\mathrm{mm}$ thick) and a protective layer of a sand-cement plaster (20 mm thick).

The distance from the ground surface to the upper part of channel was $H=1 \mathrm{~m}$ and the thickness of the channel walls was $135 \mathrm{~mm}$ (Fig. 1). The temperature of the inner surface of pipeline was $373 \mathrm{~K}$. The ambient temperature was $273 \mathrm{~K}$. The coefficient of heat transfer in all variants of the numerical analysis was $15 \mathrm{~W} /\left(\mathrm{m}^{2} \cdot \mathrm{K}\right)$.

Thermophysical characteristics of the thermal insulation and the protective layer under flooding conditions are effective (It takes into account the volume fraction of water). Table 1 contains values 
$[10,11]$ of thermophysical characteristics, which were used in the numerical investigations of thermal conditions of the system under consideration (Fig. 1).

Table 1. Thermal properties of materials.

\begin{tabular}{|c|c|c|c|c|}
\hline Material & $\begin{array}{c}\text { Protective } \\
\text { layer }\end{array}$ & $\begin{array}{c}\text { Insulating } \\
\text { layer }\end{array}$ & $\begin{array}{c}\text { Channel } \\
\text { wall }\end{array}$ & Ground \\
\hline Thermal conductivity, [W/(m·K)] & 0.87 & 0.059 & 1.54 & 1.5 \\
\hline Heat capacity, $[\mathbf{J} /(\mathbf{k g} \cdot \mathbf{K})]$ & 837 & 670 & 887 & 1150 \\
\hline Density, $\left[\mathbf{k g} / \mathbf{m}^{\mathbf{3}}\right]$ & 1750 & 206 & 2200 & 1960 \\
\hline
\end{tabular}

\section{Results of numerical simulation}

The main results of numerical modeling of thermal conditions of the system under consideration (Fig. 1) are listed in Table 2 and in Fig. 2.

Table 2. Results of numerical simulation.

\begin{tabular}{|c|c|c|c|c|c|c|}
\hline Calculation version & \multicolumn{1}{|c|}{$\begin{array}{c}Q_{0} \\
{[10]}\end{array}$} & \multicolumn{2}{|c|}{$Q$} \\
\hline $\begin{array}{c}\text { Volume fraction of water in the } \\
\text { insulation structure }\end{array}$ & \multicolumn{2}{|c|}{0.0} & 0.1 & 0.25 & 0.5 & 0.73 \\
\hline Heat loss, [W/m] & 132.29 & 134.38 & 207.16 & 274.53 & 337.29 & 370.66 \\
\hline$\frac{\boldsymbol{Q}-\boldsymbol{Q}_{0}}{\boldsymbol{Q}} \mathbf{1 0 0 \%}$ & - & 1.5 & 30.1 & 51.8 & 60.8 & 64.3 \\
\hline
\end{tabular}

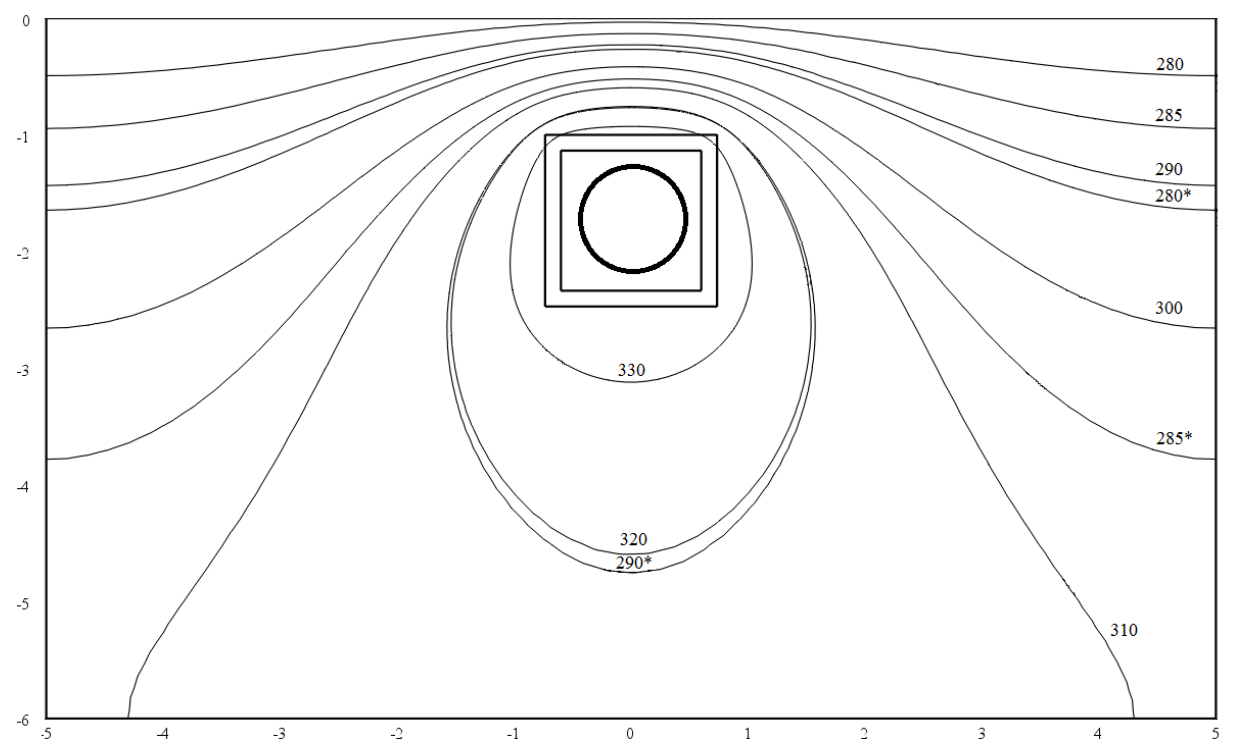

Figure 2. Temperature field of the ground in the region of an underground channel heat pipeline under flooding conditions.

Validity and reliability of the obtained results follow from tests of the methods for convergence and stability of solutions on multiple meshes, fulfillment of the energy balance conditions at boundaries of the calculation domain. The relative calculation error in all versions of the numerical 
analysis did not exceed $0.5 \%$, which is acceptable for investigations of thermal conditions of the system under consideration (Fig. 1).

Table 2 lists the results of numerical experiments of the heat loss of a heat pipeline under flooding conditions with use a convective-conductive heat transfer model $Q$ and heat loss of an underground channel heat pipeline without flooding conditions $Q_{0}[10]$.

The numerical experimental results in Table 2 allow us to make the inference about the expected increase of the heat loss of an underground channel heat pipeline with growing the volume fraction of water in the insulation structure. The heat loss of an underground channel heat pipeline $Q$ increase from 1.5 to $64.3 \%$ compared with the heat loss $Q_{0}$.

Figure 2 represent a typical temperature field of the ground in the region of an underground channel heat pipeline under flooding conditions. The superscript "*” is the temperature field from [10].

\section{Conclusion}

We have carried out numerical analysis of thermal regimes and numerical analysis of heat loss of an underground channel heat pipeline under flooding conditions with use a convective-conductive heat transfer model. It has been shown that application of the proposed approach enables comprehensive analysis of thermal regimes of the system under consideration.

\section{References}

1. B. Rezaie, M.A. Rosen, Applied Energy., 93, 2 (2012)

2. D. Magnusson, Energy Policy, 48, 449 (2012)

3. D.J.C. Hawkey, Environmental Innovation and Societal Transitions, 5, 19 (2012)

4. E. Fahlén, E.O. Ahlgren, Energy Policy, 38, 4909 (2010)

5. K. Comakli, B. Yuksel, O. Comakli, Applied Thermal Engineering, 24, 1009 (2004).

6. A. Dalla Rosa, H. Li, S. Svendsen, Energy., 36, 2407 (2011)

7. G.V. Kuznetsov, V.Yu. Polovnikov, Thermal Engineering, 55 (5), 426 (2008)

8. G.V. Kuznetsov, V.Yu. Polovnikov, J. Eng. Phys. Thermophys., 81 (2), 323 (2008)

9. G.V. Kuznetsov, V.Yu. Polovnikov, Thermal Engineering, 59 (4), 310 (2012)

10. G.V. Kuznetsov, V.Yu. Polovnikov, J. Eng. Thermophys., 20 (2), 217 (2011)

11. V.Yu. Polovnikov, E.V. Gubina, J. Eng. Phys. Thermophys., 87 (5), (2014)

12. A.L. Garcia, Numerical methods for physics (Prentice Hall, New York, 2000)

13. J. N. Reddy An Introduction to Nonlinear Finite Element Analysis (Oxford University Press, New York, 2004) 